LA-UR-99-3271

Approved for public release; distribution is unlimited.

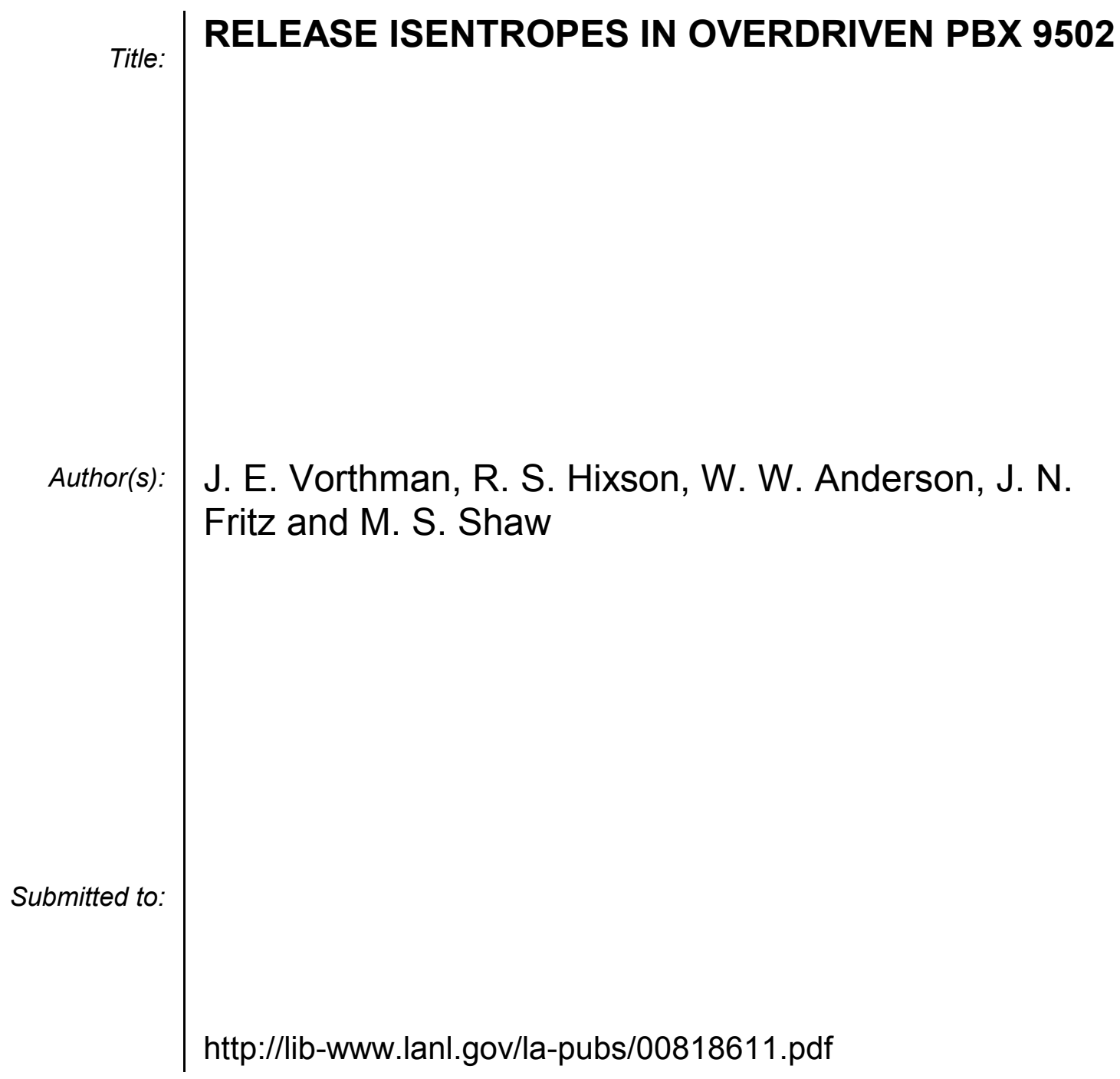

Los Alamos National Laboratory, an affirmative action/equal opportunity employer, is operated by the University of California for the U.S. Department of Energy under contract W-7405-ENG-36. By acceptance of this article, the publisher recognizes that the U.S. Government retains a nonexclusive, royaltyfree license to publish or reproduce the published form of this contribution, or to allow others to do so, for U.S. Government purposes. Los Alamos National Laboratory requests that the publisher identify this article as work performed under the auspices of the U.S. Department of Energy. Los Alamos National Laboratory strongly supports academic freedom and a researcher's right to publish; as an institution, however, the Laboratory does not endorse the viewpoint of a publication or guarantee its technical correctness. 


\title{
RELEASE ISENTROPES IN OVERDRIVEN PBX 9502*
}

\author{
J. E. Vorthman, R. S. Hixson, W. W. Anderson, J. N. Fritz and M. S. Shaw
}

Los Alamos National Laboratory, Los Alamos, New Mexico 87545 USA.

\begin{abstract}
Experiments have been done to obtain product isentropes of the plastic bonded TATB explosive PBX 9502. A thin aluminum flyer was thrown at an explosive disk so as to generate a state in excess of the Chapman-Jouguet pressure. Because the flyer is thin, the shock wave is followed by a release wave that lowers the pressure in the explosive products. The explosive disk was backed by a LiF tamper/window. Particle velocity at the explosive - window interface was measured with a velocity interferometer (VISAR) and measured particle velocity records were used to construct isentropes. Unexpected behavior is seen in the shape of the isentropes. Experimental details and the resulting isentropes will be presented.
\end{abstract}

\section{INTRODUCTION}

PBX 9502 is a TATB based plastic bonded high explosive (1). In this paper we present the results of experiments done to obtain high-pressure isentropes of the PBX 9502 explosive products. Here, high pressure means pressure in the explosive in excess of its Chapman-Jouguet (CJ) pressure (2). Overdriven detonation waves reduce reaction zone effects by speeding up reaction rates. A typical experiment consisted of a thin aluminum impactor, an explosive disk, and a LiF window / anvil. When the aluminum flyer hit the disk of PBX 9502, the impact generated a high-pressure shock wave in the explosive and, because the flyer was thin, the shock wave was followed by a release wave that subsequently lowered the pressure in the explosive products. Particle velocity at the interface between the explosive and $\mathrm{LiF}$ window was measured with a VISAR (3). The VISAR record was later used to construct a high-pressure isentrope for the explosive products. Construction of the isentrope is complicated by the fact that the LiF window has slightly higher impedance than the explosive products. VISAR records from the higher-pressure experiments show a different character than those from lower pressure experiments and this leads to differ-

\footnotetext{
* This work supported by the US Department of Energy
}

ences in the respective isentropes. Such differences in the isentropes support the hypothesis of phase changes (4) and/or chemical reactions in the detonation products themselves. Several experiments were done to insure that the different wave-profile shapes were not an experimental artifact. Details of the experiments are presented followed by a short description of how the isentropes are constructed, the resulting isentropes are shown, and finally, conclusions will be presented.

\section{THE EXPERIMENTS}

An explosive plane wave lens plus booster was used to accelerate the aluminum flyer. The type and size of the booster explosive, as well as assembly dimensions were varied to produce different flyer velocities. Overall flyer diameter was about $30 \mathrm{~cm}$ but only the center part (about $15-\mathrm{cm}$ ) of the flyer was used to collect data. The large flyer was used so that near the center, flyer bow was minimized to the point where it does not affect the data. A "Ub" assembly (see Fig. 1) located adjacent to the explosive sample was used to measure flyer velocity. The flyer strikes the two levels of the Ub assembly at different times and so there are two distinct light 


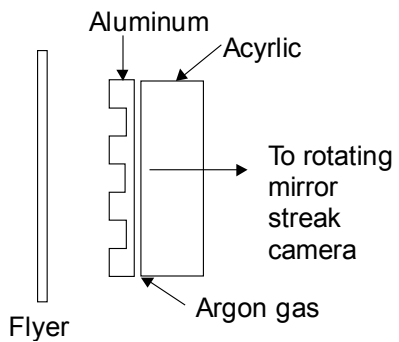

FIGURE 1. Ub assembly

flashes when the argon gas is squeezed between the aluminum and acrylic parts of the Ub assembly. The light flashes are recorded on film using a rotatingmirror streak camera. Flyer velocity measured with this technique is accurate to between 1 and $2 \%$. Flyer bow causes the flyer to hit one part of the Ub first and this is also visible on the film.

Nominal sample dimensions are $76.2 \mathrm{~mm}$ in diameter by $12.7 \mathrm{~mm}$ thick. Measured sample density was $1.890 \pm 0.002 \mathrm{~g} / \mathrm{cm}^{3}$. A $17-\mu \mathrm{m}$ aluminum foil with a diffuse surface was glued between the sample and the LiF window. Particle velocity of this foil was measured with a VISAR. The LiF window was nominally $50.8 \mathrm{~mm}$ in diameter and $25.4 \mathrm{~mm}$ thick. $\mathrm{LiF}$ was chosen as a window because it is well calibrated as a VISAR window (5) and because it is a close match in shock impedance to the explosive products and to the aluminum foil. Data was recorded until either release waves from the edge of the sample reached the center of the sample or the shock wave in the LiF window reached the end of the window.

Measured interface velocities from the different experiments are shown in Fig 2. Experimental parameters are listed in Table 1. The major features of the waveforms are (a) a shock jump to a constant particle velocity and (b) a more gradual decrease in particle velocity as the pressure in the sample drops.
The width of the region of constant particle velocity was adjusted by varying the thickness of the aluminum flyer. Note that the lower pressure experiments show an initial overshoot in particle velocity (and pressure). The chemical reaction(s) which transform the solid explosive to gaseous products takes a finite time and here we are able, in the overshoot in the lower pressure experiments, to resolve part of the transformation. Also notable is the fact that the higher-pressure experiments show waveforms that are not as smooth as the waveforms in the lower pressure experiments (in the region of decreasing particle velocity).

Much work was done prove that these "wiggles" in the high pressure data are due to real properties of the explosive sample and are not experimental artifacts. Figure 3 shows two waveforms from experiments where the explosive target is replaced with a disk of aluminum of the same size. The waveforms from the aluminum experiments clearly show an elastic release wave (also visible in the first part of the explosive sample waveforms) followed by a very smooth, wiggle free, decrease in particle velocity. To further assure that the wiggles are not an artifact of VISAR data reduction, most of the experiments were done with two VISARs on the same experiment. The two VISARs had different etalon lengths and so the raw data from the two VISARs are very different. The reduced data from the two different VISARs typically agree within $0.5 \%$.

\section{CALCULATING ISENTROPES}

Turning the measured particle velocity waveforms into isentropes is complicated by the fact that the shock impedance of the LiF window is slightly larger that the impedance of the explosive products. If the impedance of the explosive products were equal to or higher than that of the window, the re-

TABLE 1. Shot Description

\begin{tabular}{|c|c|c|c|c|}
\hline Shot \# & $\begin{array}{c}\text { Flyer } \\
\text { thickness } \\
\text { (mm) }\end{array}$ & $\begin{array}{c}\text { Flyer } \\
\text { Velocity } \\
\text { (km/s) }\end{array}$ & $\begin{array}{c}\text { Sample } \\
\text { thickness } \\
\text { (mm) }\end{array}$ & $\begin{array}{c}\text { Impact } \\
\text { pressure } \\
\text { (GPa) }\end{array}$ \\
\hline 4F63 & 4.704 & 5.79 & 12.711 & 55.5 \\
4F65 & 3.206 & 4.85 & 12.708 & 43.7 \\
3R68 & 4.708 & 5.01 & 12.702 & 45.6 \\
3R69 & 4.717 & 6.45 & 12.704 & 64.7 \\
4F112 & 6.378 & 3.68 & 12.706 & 31.0 \\
4F130 & 4.711 & 5.86 & 12.701 & 56.4 \\
4F131 & 4.699 & 4.23 & 12.686 & 36.7 \\
\hline
\end{tabular}




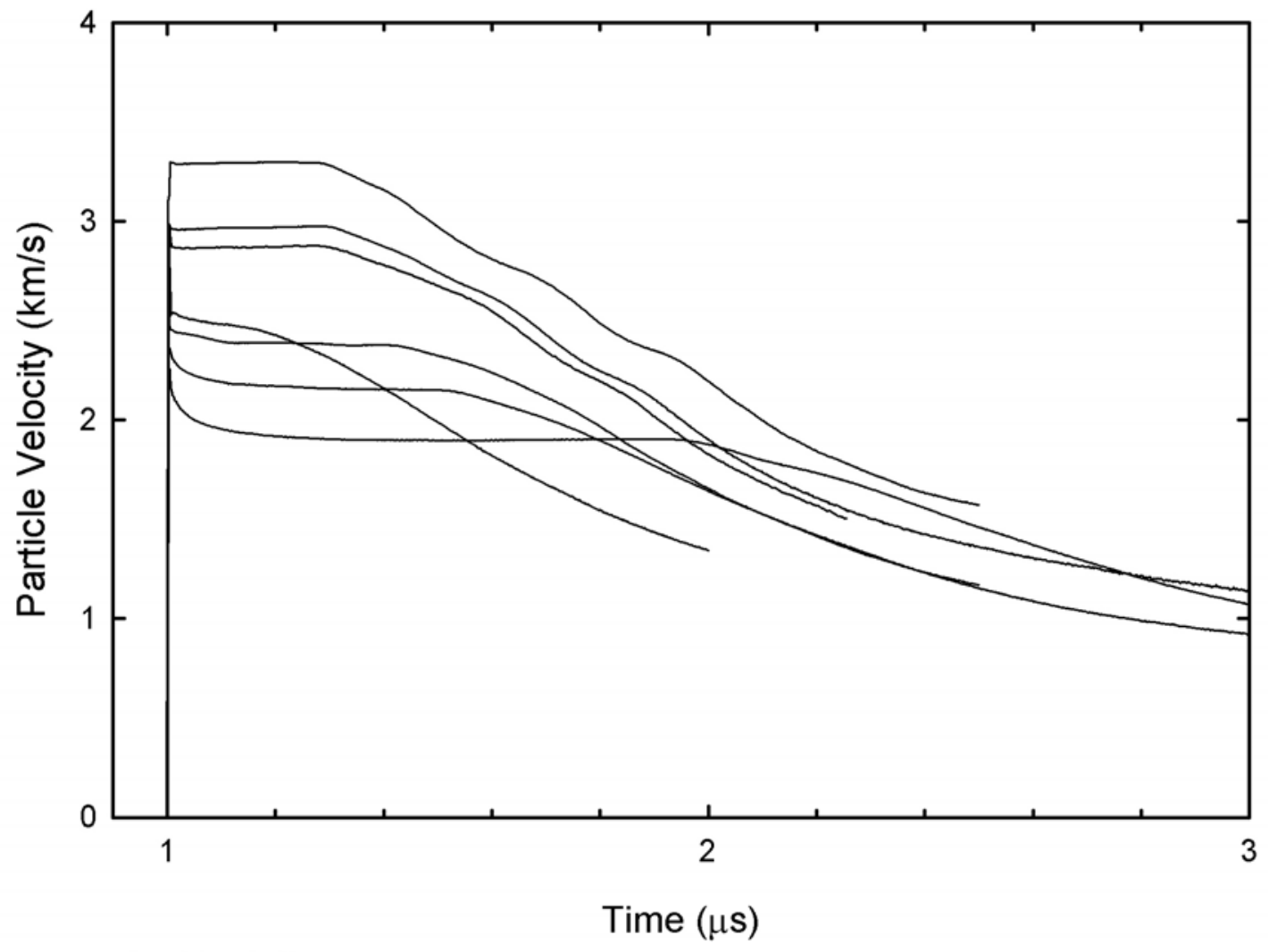

Figure 2. Measured Particle Velocities.

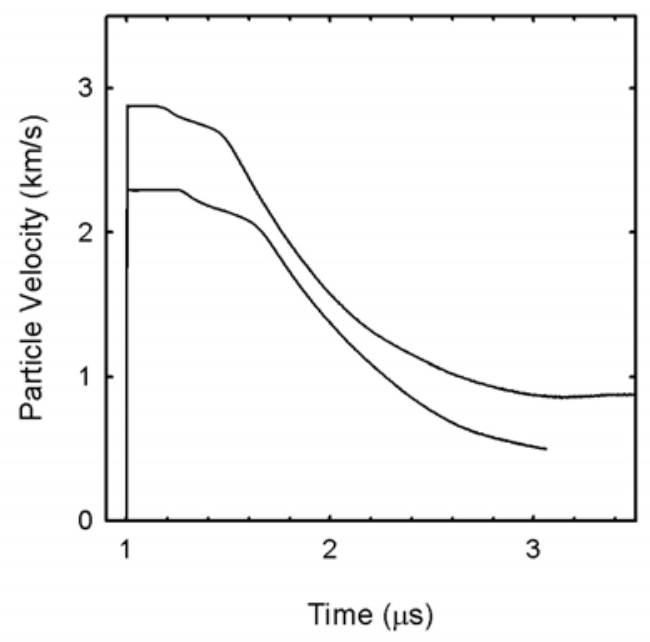

Figure 3. Aluminum Targets.

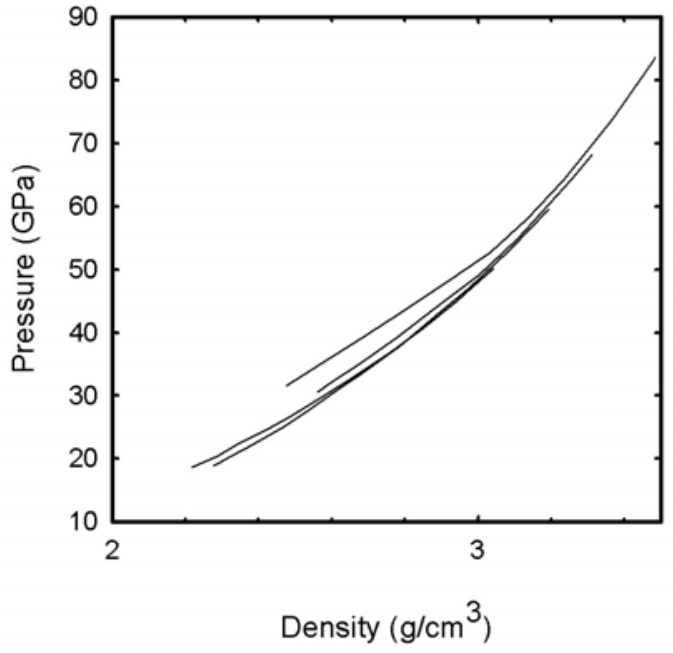

Figure 4. Selected Reference Isentropes. 
lease would lie on a single isentrope. To reduce the data, a functional form was assumed for the equation of state (EOS) of the detonation products. Adjustable parameters in this EOS were varied until a hydrocode could reproduce the measured waveforms. The usual Mie-Grüneisen form of the EOS was used in the hydrocode:

$$
P(\rho, E)=P_{R}(\rho)+\rho \gamma\left(E-E_{R}(\rho)\right)
$$

where $\rho \gamma$ is a constant, $P_{R}(\rho)$ is a reference isentrope and $E_{R}$ is obtained by integration of the reference isentrope. The form of the reference isentrope is

$$
P_{R}(\rho)=f(\rho) \rho^{\gamma_{0}}
$$

where $\gamma_{0}$ is a constant approximately equal to the adiabatic $\gamma_{\mathrm{s}}$ in the region of interest and $f(\rho)$ is a piecewise linear function

$$
f(\rho)=f_{i}+\left(f_{i+1}-f_{i}\right) \frac{\rho-\rho_{i}}{\rho_{i+1}-\rho_{i}} .
$$

Using the above EOS, calculations were done with the hydrodynamics code, Hydrox (6). In an automated process, the parameters $f_{i}$ were adjusted to give the best match of the calculated particle velocity to the measured particle velocity (as well as the measured Hugoniot). Figure 4 shows the resulting reference isentropes for several waveforms. Figure 5

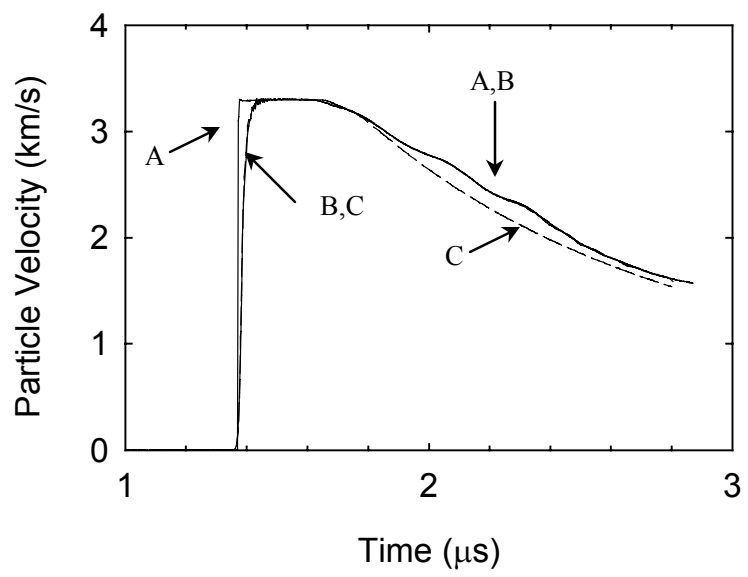

Figure 5. Comparison of data (A) to calculation using fitted reference isentrope (B) and calculation using reference isentrope derived from sound speed and Hugoniot data (C). shows how well a calculation using the preferred $f_{i}$ reproduces the measured particle-velocity. Also shown in Fig. 5 is an example of a hydrocode calculation where only sound speed and Hugoniot data are used to generate the reference curve.

\section{DISCUSSIONS AND CONCLUSIONS}

The reference isentropes shown in Fig. 4 show behavior not yet seen in other explosives. For the higher-pressure experiments, there is a distinct change in slope of the isentropes near $3 \mathrm{~g} / \mathrm{cm}^{3}$. This change in slope leads to a marked separation of isentropes (comparing lower initial-shock-pressure experiments to higher-pressure experiments). One explanation of this type of behavior is additional chemical reactions that do not occur at lower pressure. This possibility is discussed in greater detail by Shaw (7).

\section{REFERENCES}

(1) Gibbs, T. R. and Popolato, A., LASL Explosives Property Data, University of California Press, Berkeley and Los Angeles, California, 1980, pp. 120129.

(2) Fickett, W. and Davis, C. D., Detonation, , University of California Press, Berkeley and Los Angeles, California, 1979, pp. 19-20.

(3) Hemsing. W. F., Velocity sensing interferometer (VISAR) modification, Rev. Scient. Instr., 50(1), $73-$ 78, (1979).

(4) Tang, P. K., Anderson. W. W., Fritz, J. N., Hixson, R. S., and Vorthman, J. E., "A Study of the Overdriven Behaviors of PBX 9501 and PBX 9502, Eleventh Symposium (International) on DETONATION, in press.

(5) Wise, J. L. and Chhabildas, L. C., "Laser Interferometer Measurements of Refractive Index in ShockCompressed Materials", in Shock Waves in Condensed Matter, edited by Y. M. Gupta, AIP Conference Proceedings, New York, 1996, pp.441-454.

(6) Shaw, M. S. and Straub G. K., HYDROX: A OneDimensional Lagrangian Hydrodynamics Code, Technical Report No. LA-8642-M, Los Alamos National Laboratory, 1981.

(7) Shaw, M. S., A Theoretical Equation of State For Detonation Products with Chemical Equilibrium Composition of the Surface of Small Carbon Clusters, these proceedings. 